\title{
Improving Interactive Video in Ad-hoc Networks Using Path Diversity
}

\author{
Ahmed Abd El Al, Tarek Saadawi, Myung Lee \\ Dept. of Electrical Engineering, City College and Graduate Center of University of New York \\ aabdelal@ieee.org, \{saadawi,lee\}@ccny.cuny.edu
}

\begin{abstract}
*
In this paper, we propose a mechanism that combines retransmission-based error control with path diversity, to provide different levels of protection to interactive video in ad-hoc networks. The mechanism factors in the importance of the retransmitted packets to the reconstructed video quality as well as the endto-end latency constraints to minimize the overhead and maximize the reconstructed video quality at the receiver. Simulation results show that the proposed retransmission mechanism maintains the video quality under different loss rates and with less overhead compared to error control methods that depend on controlling the intra-update rate. In addition, the mechanism is shown to be more robust to wireless losses than schemes that combine layered coding with path diversity.
\end{abstract}

\section{Introduction}

Video transmission in ad-hoc networks is a challenging problem, due to the high bit error rates which can range from single bit errors to burst errors or even intermittent loss of the connection. The high error rates are due to multi-path fading, which characterizes mobile radio channels, while the loss of the connection can be due to the mobility in such networks. In addition, designing the wireless communication system to mitigate these effects can be complicated by the rapidly changing quality of the radio channel.

All common video coding schemes, including standards like H.263 and MPEG, are designed to achieve high compression efficiency on expense of the error resilience. The coding efficiency in these schemes

\footnotetext{
* Prepared through collaborative participation in the Communications and Networks Consortium sponsored by the U.S. Army Research Laboratory under the Collaborative Technology Alliance Program, Cooperative Agreement DAAD19-01-2-0011.
}

is achieved by using motion-compensated prediction to exploit the redundancy between successive frames of a video sequence. A motion-compensated video sequence consists of two types of video frames: intraframes (I-frames) and inter-frames (P- or B-frames). Iframe is encoded by only removing spatial redundancy present in the frame. P-frame is encoded through motion estimation using preceding I- or P-frame as a reference frame. B-frame is encoded bi-directionally using the preceding and succeeding reference frames. For each image block in an inter-frame, motion estimation finds a closely matching block within its reference frame, and generates the displacement between the two matching blocks as a motion vector. The pixel value differences between the original interframe and its motion-predicted frame are encoded along with the motion vectors. Removing the temporal redundancy within the stream poses a severe problem, namely error propagation (or error spread), where errors due to a packet loss in a reference frame propagate to all of the dependent frames leading to perceptible visual artifacts.

Error propagation can be controlled by more frequently adding intra-frames (which are coded temporally independently). However, the ratio of the compression efficiency of an intra-frame over an interframe is as large as 3 to 6 times. Increasing the frequency of intra-frames could increase the bandwidth requirement too much for video transmission over a bandwidth-constraint network. Hence, by adjusting the amount of Intra-coding, error robustness can be traded off against coding efficiency [1].

The widely varying error conditions in wireless channels limit the effectiveness of classic Forward Error Correction (FEC), since a worst-case design would lead to a prohibitive amount of redundancy. Although retransmission-based error recovery requires minimal network bandwidth and processing cost than FEC based error control methods, it is often considered inappropriate for interactive video, because of the limited playout delay at the receiver, which limits the number of admissible retransmissions [2]. 
In this paper, we propose an error control mechanism for interactive video applications in mobile ad-hoc networks. The mechanism extends retransmission-based error control with redundant retransmissions on diverse paths provided by the adhoc routing protocol between the sender and receiver. The mechanism factors in the importance of the retransmitted packets to the reconstructed video quality as well as the end-to-end latency constraints to minimize the overhead and maximize the quality at the receiver. We compared the proposed mechanism to error control methods that depend on intra-frame updates, as well as mechanisms that combine layered coding with multipath transport. Simulation results indicate that the proposed mechanism performs significantly better than reference frame update schemes in terms of perceived quality measured at the receiver as well as the transmission overhead. It is also more robust to wireless losses than layered coding schemes under comparable path conditions.

This paper is organized as follows. Section 2 provides a review for related works. The proposed mechanism is presented in Section 3. Section 4 discusses the mechanism implementation. We present experimental results and performance evaluation in Section 5. Finally, conclusions are outlined in Section 6.

\section{Related Work}

Error control mechanisms can be classified into four types, namely, Forward Error Correction (FEC), retransmission, error-resilient encoding, and error concealment [3].

The principle of FEC is to add extra (redundant) information to a compressed video bit-stream so that the original video can be reconstructed in presence of packet loss. The redundancy added in FEC-based approaches leads to increased bandwidth requirements. The FEC-based approaches are designed to overcome a predetermined amount of channel losses. If the losses are less than the threshold, then the transmitted data can be perfectly recovered from the received lossy data. However, if the losses are greater than the threshold, then only a portion of the data may be recovered, and depending on the type of FEC used, the data may be completely lost [4]. Due to the fact that wireless channel is non-stationary, and the channel bit error rate varies over time, in most cases the FEC is either over designed and therefore inefficient, or underdesigned and therefore ineffective. Furthermore, in mobile wireless networks, the assumption that burst length is short does not hold as long burst losses can be common due to fading and channel interference [5].

Unlike FEC, which adds redundancy regardless of correct receipt or loss, reference [6] proposes retransmission-based error control schemes, such as Automatic Repeat Request (ARQ), for real time data. Retransmission-based schemes resend only the packets that are lost, thus they are adaptive to varying loss characteristics, resulting in efficient use of network resources. However, retransmission schemes are limited by the receiver's playout delay as well as the Round Trip Time (RTT) [7]. Reference [8] proposes an error recovery technique that combines FEC and retransmission to solve the error propagation problem. Instead of preventing display errors from occurring, the mechanism uses late repair packets to stop error propagation, which allows more time for packet repairs and masks out delays in repairing lost packets.

Error-resilient encoding schemes address loss recovery from the compression perspective. Specifically, they attempt to prevent error propagation or limit the scope of the damage (caused by packet losses) on the compression layer. Examples of errorresilient encoding are Multiple Description Coding (MDC) and Layered Coding (LC). MDC generates multiple equally important, and independent substreams, also called descriptions. Each description can be independently decoded and is of equal importance in terms of quality, i.e. there is no decoding dependency between any two of the descriptions. When the decoder receives more descriptions, the quality can be gradually increased no matter which description is received [9]. Reference [10] examines the effectiveness of combining MDC and Multiple Path Transport (MPT) for video and image transmission in a multihop mobile radio network. The primary inefficiency in MDC is that in order to guarantee an acceptable quality with a single description, each description must carry sufficient information about the original signal. This implies that there will be overlap in the information contained in different descriptions. Obviously, this will reduce the coding efficiency compared to the conventional Single Description Coding (SDC). In addition, although more combined descriptions provide a better visual quality, a certain degree of correlation between the multiple descriptions has to be embedded in each description, resulting in further reduction of the compression efficiency. LC generates one base-layer bitstream and several enhancement-layer bitstreams. The base-layer can be decoded to provide a basic video quality while the enhancement-layers are mainly used to refine the quality of the video that is reconstructed from the base-layer. However, if the base-layer is 
corrupted, the enhancement-layers become useless, even if they are received perfectly. Reference [11] proposes a scheme for reliable transmission of video over bandwidth limited ad-hoc networks. A raw video stream is layer coded and the base layer and the enhancement layer packets are transmitted separately on two disjoint paths. The base layer packets are given higher protection than enhancement layer packets. As will be shown in our performance study, the quality of the reconstructed video in this approach depends on the condition of the path that carries the base-layer packets. This will cause the video quality to fluctuate with the error characteristics of a single path. Even a very small loss rate on the base layer will make the layered coder less desirable.

Error concealment is a post-processing technique used by the decoder. It depends on the smoothness property of the images as well as that the human eye can tolerate distortion in high frequency components than in low frequency components [2]. Reference [12] shows that detectible artifacts can still exist after the error concealment, and that the degree of these artifacts depends on the amount of lost data, the type of the stream and the effectiveness of the concealment algorithm. High-quality concealment algorithms require substantial additional computation complexity, which is acceptable for decoding still images but not tolerable in decoding real-time video. In addition, the effectiveness of concealment depends on the amount and correct interpretation of received data, thus concealment becomes much harder with the bursty losses in wireless channels.

\section{Prioritized Retransmission Over Diverse Paths}

The ability to successfully decode a compressed bitstream with inter-frame dependencies depends heavily on the receipt of reference frames, and to a lesser degree on dependent frames. Thus, we propose a mechanism to provide adaptive end-to-end unequal error protection for packets belonging to different frames, without sacrificing the timely-delivery requirement for interactive video. We achieve the unequal protection through redundant retransmissions over diverse paths between the sender and receiver. Due to the statistical independence of the packet loss events over different paths, by re-transmitting the packets over separate paths, we are maximizing the probability that at least one packet is received errorfree, in least number of retransmissions. This behavior is required especially for interactive video, with limited playout delay at the receiver. The scheme is adaptive in the sense that the retransmission overhead will only be added when there is loss in the stream, and the degree of the overhead is proportional to the importance of the lost packets. To ensure in-time delivery of retransmitted packets, and to prevent re-transmitting expired packets, the retransmission is controlled by the packet lifetime, as well as estimate(s) of the path(s) delay. As will be shown in our performance study in Section 5, the proposed prioritization scheme can significantly limit the effect of error propagation and improves the quality of received video, under a limited playout delay.

\subsection{Paths Diversity in Ad-hoc Networks}

Due to the mesh structure of ad-hoc networks, most routing protocols can provide multiple loop-free and maximally disjoint paths [13][14]. In addition, nodes can employ multiple channels, using multiple frequencies or time slots, to allow concurrent data transmission [15]. Redundant retransmission over diverse paths increases the probability that the packet get received in less number of retransmissions. With a network loss rate $l$, the error rate can be reduced to

$$
\text { Error Rate }=l^{1+\sum_{i=1}^{L} M_{i}}
$$

where $L$ is the maximum number of retransmission trials, which is typically determined by the initial playout delay in the receiver as well as the round-trip delay. $M_{i}$ is the number of retransmission copies during the $i^{\text {th }}$ retransmission, which depends on the importance of the retransmitted data to the reconstructed video quality. The maximum number of copies $\operatorname{MAX}\left(M_{i}\right)$ is equal to the number of available paths between the sender and receiver.

\subsection{Packet Prioritization}

The priority for each data unit in the stream is determined by the application. Thus in the context of motion compensated coding, the application can assign higher priority for I-frames data, than P- or B- frames data. Also $\mathrm{P}$-frames might be assigned varying priority levels, since P-frames that are closer to the preceding Iframe are more valuable for preserving picture quality than later P-frames in the group of pictures (GOP). The prioritization scheme can also be applied on the macroblock basis in case of coding schemes which provides the encoder with the flexibility to select the coding mode, i.e. intra or inter coding, on the macroblock level [16]. 


\section{Implementation}

We implemented the proposed mechanism as a sublayer above Real Time Protocol (RTP) [17]. Figure 1 shows the system architecture. We refer to this sublayer as Multiple Path-RTP (MP-RTP). MP-RTP is responsible for:

1) Maintaining the reliability level and the lifetime for each packet, as well as implementing delay constrained retransmission,

2) Monitoring the status of the available paths, and selecting the suitable path(s) for packet retransmission.

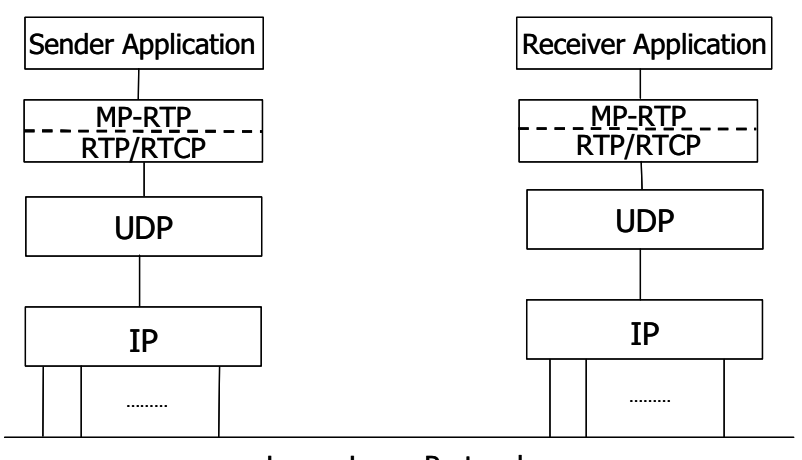

Lower Layer Protocols

Figure 1. System architecture

For each video frame, the sending application assigns a priority level, which is based on the frame's importance to the reconstructed video quality. For example, I-frames can be assigned higher reliability level than P- or B- frames. Also P-frames can be assigned varying reliability levels based on their location in the GOP. In addition, the sending application calculates the lifetime for each video frame $N, T_{L}(N)$, as follows:

$$
T_{L}(N)=T_{R}(N)+D_{S}
$$

where $T_{R}(N)$ is an estimate for the rendering time of frame $N$ at the receiver, and $D_{S}$ is a slack term to compensate the inaccuracies in estimating the OneWay-Delay $(O W D)$ from the sender to the receiver, as will be discussed later, as well as the receiver's processing delay. Assuming that there is no compression and/or expansion of total display time at the receiver, the rendering time for frame $N, T_{R}(N)$, is calculated as follows:

$$
T_{R}(N)=T_{0}+T_{D}+N / R
$$

where $T_{0}$ is the video session initiation time, $T_{D}$ is the receiver's playout delay, which determines the rendering time for the first frame in the sequence. Playout delay can be obtained from the receiver during the session initiation. $R$ is the frame rate.

As the MP-RTP sub-layer receives a frame it fragments it, if required, into multiple packets, then RTP headers are added and the packets are sent to the receiver. In addition, a copy of each packet is kept in a retransmission buffer, along with its lifetime and priority. Typically, all the packets within one frame will have the same lifetime and priority. MP-RTP clears packets from the retransmission buffer, as it receives the Real Time Control Protocol-Receiver Reports (RTCP-RR), which are sent regularly from the receiver, indicating the highest sequence number received, as well as other information regarding the quality of the received stream [17]. Initially, packets are sent on a primary path with the receiver, selected by the sender during the session initiation. The transmission rate on the primary path is dynamically adjusted based on the on the receiver's feedback.

The MP-RTP at the receiver is responsible for sending retransmission requests to the sender as soon as it detects a missing packet. The format of the retransmission request, shown in figure 2, is similar to RTCP-RR, except that it is extended to include the 32 bits sequence number of the missing packet. As the retransmission request is susceptible to losses, MPRTP retransmits these reports on multiple paths to the sender.

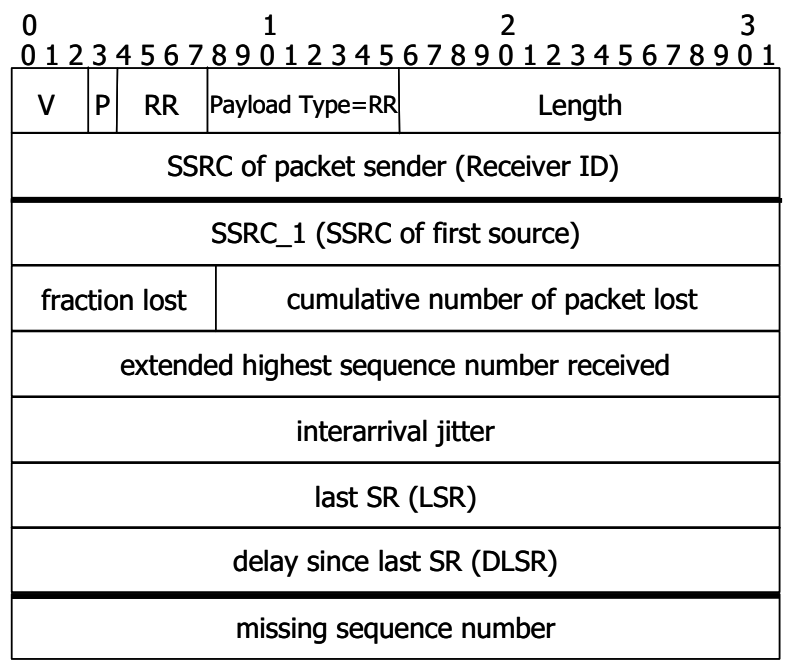

Figure 2. Extended RTCP-RR to include the missing sequence number 


0
01234567890123456789012345678901
\begin{tabular}{|l|l|l|l|}
\hline Version & Padding & Payload Type=HB & Length \\
\hline \multicolumn{4}{|c|}{ time stamp } \\
\hline
\end{tabular}

(a)

0
01234567890123456789012345678901
\begin{tabular}{|l|l|l|l|}
\hline Version & Padding & Payload Type=HB-ACK & Length \\
\hline \multicolumn{3}{|c|}{ time stamp } \\
\hline
\end{tabular}

(b)

Figure 3. (a) Heartbeat packet (b) Heartbeat acknowledgement

MP-RTP uses Heartbeat packets, shown in figure 3.a., to maintain an estimate for the RTT of the available paths. Each heartbeat packet includes a time stamp indicating the transmission time. The MP-RTP at the receiver responds to the heartbeat packet by sending a Heartbeat-Acknowledgment packet, shown in figure 3.b, on the same path from which the heartbeat was received. The heartbeat-acknowledgement includes a copy of the timestamp in the corresponding heartbeat packet. The RTT estimates are calculated using a smoothed average of the current and previous measurements. These estimates are used to obtain an approximation for the paths $O W D$, i.e., $O W D \approx R T T$, 2. The application can compensate the inaccuracies in the $O W D$ approximation as it assigns the frames lifetime, as shown in equation 2. In addition, MP-RTP uses the RTT estimates to switch the primary path, which can break due to the mobility in the network. To minimize the interruption for the interactive video session, as the primary path $R T T$ increases beyond a certain threshold, MP-RTP sets the alternative path with the shortest RTT to be the primary path. The switching threshold can be based on the maximum delay allowed for the interactive video application. Currently, we are using a fixed value for the switching threshold. In future work, we are planning to investigate techniques to dynamically adapt the value of the switching threshold.

As soon as the sender receives a retransmission request, it performs the retransmission algorithm shown in figure 4.
Let $T_{c}$ be the current time at the sender, $T_{L}(j)$ is the lifetime for frame $j$

if (lost packet belongs to low priority frame $j$ ) if $\left(T_{c}+\min \left(O W D_{i}\right)<T_{L}(j)\right)$ where path $i \varepsilon\{1, N\}$

Retransmit on path $i$

else

for all paths $i$

if $\left(T_{c}+O W D_{i}<T_{L}(j)\right)$

Retransmit on path $i$

if (packet cannot be retransmitted)

Discard packet

Notify upper layer to stop the prediction loop

\section{Figure 4. Redundant retransmission algorithm}

By controlling the retransmission through the frames lifetime, as well as estimate(s) of the path(s) delay, MP-RTP prevents retransmission of expired packets while trying to meet the frames lifetime constraint. If retransmitted the packet will not be received before the rendering time for the frame to which it belongs, the packet is discarded and the upper layer application is notified about the dropped packet to allow the encoder to utilize schemes, such as error tracking, that stop the prediction loop to limit the error propagation. Error tracking allows the encoder to reconstruct the resulting error distribution, due to the lost packet, in the next frame to be encoded, then it encodes the blocks contained in this region in intra-mode [12].

\section{Performance Analysis}

We implemented the proposed mechanism in the OPNET simulation and modeling tool [18]. The network topology used in our performance study, is shown in figure 5. We simulated a Multi Path Transport (MPT) system, with configurable number of paths between the sender and receiver. For simplicity we assumed that the paths are identical in terms of available bandwidth and each path has three wireless hops, each modeled by the two-state model Markov model, with identical parameters. 


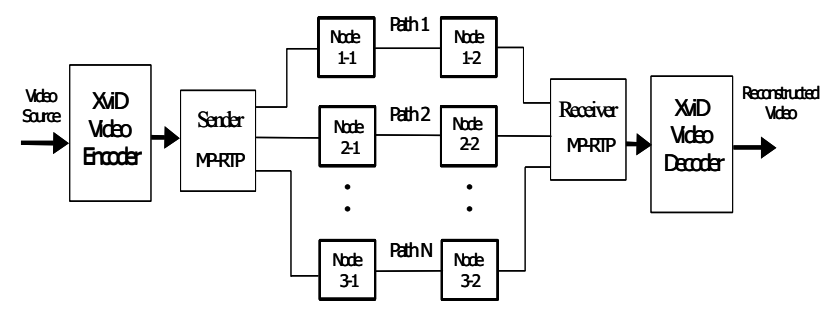

Figure 5. Simulation topology

The two state model, shown in figure 6, is often referred to as Gilbert channel model, and is used to simulate the bursty packet loss behavior in wireless networks [19]. This simple model has been shown to be able to effectively capture the bursty packet loss behavior of the wireless channels. The two states of this model are denoted as Good $(G)$ and Bad $(B)$. In state $G$, packets are received correctly whereas, in state $B$, packets are assumed to be lost. This model can be described by the transition probabilities $p$ from state $G$ to $B$ and $q$ from state $B$ to $G$. The average Packet Loss Rate $(P L R)$ is:

$$
\text { Average } P L R=\frac{p}{p+q}
$$

We vary the error characteristics for channel $i$ by appropriately controlling the channel Good and Bad durations, according to an exponential distributions with averages $p_{i}$ and $q_{i}$, respectively. Delay for channel $i$ is modeled by an exponential distribution with the mean delay $D_{i}=10 \mathrm{msec}$. In addition, the radio channels are operating at $2.0 \mathrm{Mbps}$. We set the path maximum transfer unit (MTU) of 500 bytes for all the paths. The heartbeat interval is set to $150 \mathrm{msec}$. The receiver's playout delay was limited to $100 \mathrm{msec}$., to represent an interactive video application. The switching threshold, discussed in Section 4, was set to 300 msec. This value was selected because given the channel delays and the playout delay at the receiver, having the RTT of the primary path higher than this threshold will ensure that all the frames will arrive late at the receiver than their rendering time and will be discarded. In order to study the effect of wireless channel, we have set the parameters such that the losses occurs only due to channel errors and not due to, for example, buffer overflows or IP service rates.

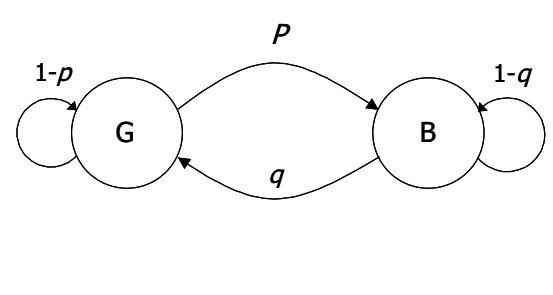

\section{Figure 6. A two-state Markov model to simulate burst packet losses}

To generate the video sequence used in our simulation, we used open source XviD MPEG-4 compliant video codec [20]. Forty seconds of a video sequence are encoded at $15 \mathrm{f} / \mathrm{s}$ and transmitted which results in a sequence of 600 frames. The frame resolution is quarter common intermediate format (QCIF, $176 \times 144$ pixels), which is the most common format at low bit rates, and the coding rate is $200 \mathrm{Kbps}$. The image distortion is computed using the average peak signal-to-noise ratio (PSNR) of decoded frames over the original frames. Without transmission losses, the average PSNR of the decoded frames for the video sequence used in our performance study is $38 \mathrm{~dB}$.

After obtaining a transmission trace of a video sequence, we run the decoder on the trace to measure the image distortion due to packet losses, using the PSNR. In order to generate statistically meaningful quality measures, for each simulation scenario we repeated the experiment ten times with different seeds. The presented PSNR values are the average of the ten experiments.

In our performance study we set the application to choose I-frames and half of the P-frames starting from the I-frame in a GOP to be high priority frames, while other frames are set to low priority frames. As will be shown in our analysis later, this setting is a compromise between video quality and the transmission overhead.

\subsection{Effect of Packet Loss Rate on Video Quality}

To examine the effect packet losses on the reconstructed video quality, we tested MP-RTP using two diverse paths, namely path- 0 and path- 1 . Path- 0 was selected as the primary path during the video session initiation. The channel average packet loss rates for path-0 and path- 1 were set to 0.3 and 0.2 respectively. 


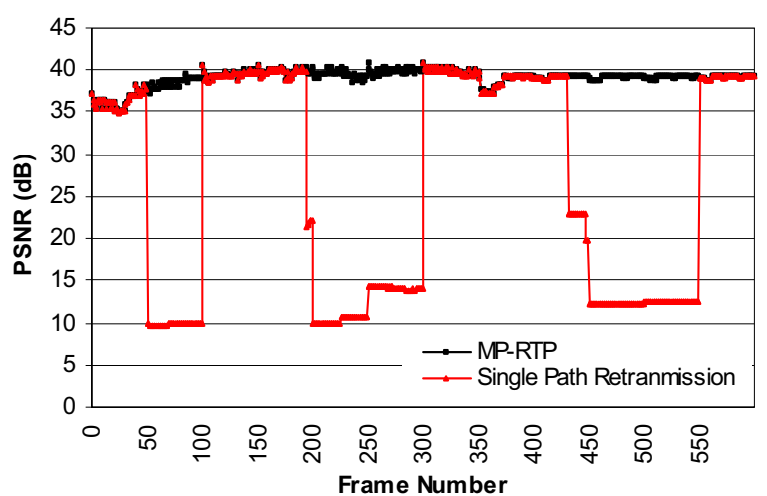

Figure 7. PSNR versus frame number

We set the encoder so that the I-frame update period, i.e. interval between two consecutive I-frames, equal 3 seconds. Figure 7 shows the PSNR for each frame in the video sequence. For comparison we repeated the experiment using single path retransmission scheme, where missing packets are retransmitted on a randomly selected path between the sender and receiver. The figure shows that the redundant retransmission in MP-RTP maintains the video quality at high packet loss rates. On the other hand, with the single path retransmission scheme, the video quality dropped for long durations due to packet losses in reference frames then the error propagates to the following dependent frames up to the next I-frame. Due to the limited playout delay at the receiver, the sender cannot keep retransmitting the lost packet, as it will be discarded by the receiver.

The average PSNR over the whole sequence versus different channel average packet loss rates for the primary path, i.e. path- 0 , is shown in figure 8 . The channel average packet loss rate for path-1 is kept equal to 0.2 . We repeated the experiment with different I-frame update periods. For MP-RTP we set the Iframe update period to 3 seconds. The figure shows that, the single path retransmission scheme achieves a similar performance to MP-RTP only when the I-frame frequency is increased more than three times to one every 15 frames. As the I-frames have larger sizes than $\mathrm{P}$ - and B-frames, an increased I-frame frequency for the same bit rate translates to reduced video quality since bits are now wasted to code I-frames. If the Iframe frequency is set to one in 45 frames for the single path case, the video quality deteriorates rapidly. This is due to error propagation from reference frames to dependent frames.

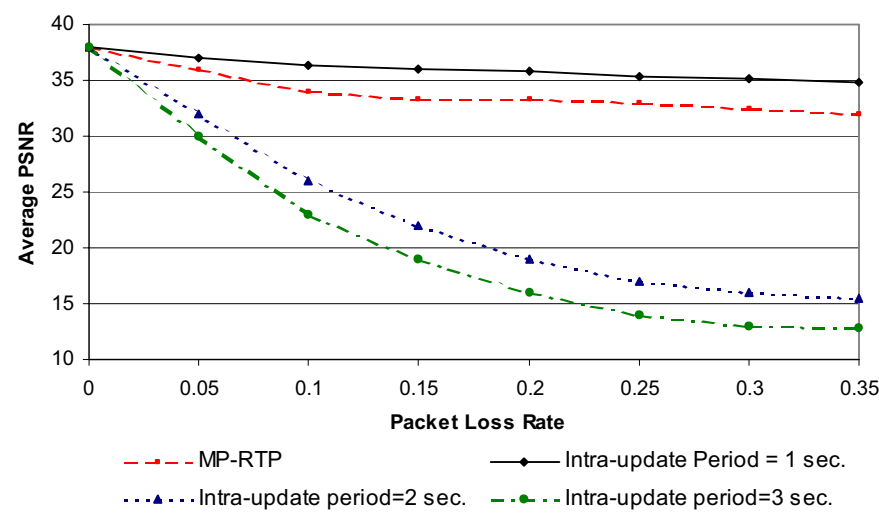

Figure 8. Average PSNR versus packet loss rate

Single path retransmission cannot limit the error propagation due to the high packet loss rate and the bounded delay for interactive video. On the other hand, the redundant retransmissions over diverse paths in MP-RTP prevent the error propagation as it ensures that at least one copy of the retransmitted packet will be received in time.

\subsection{Redundant Retransmission Overhead}

We compared the overhead of MP-RTP, due to the redundant retransmissions and heartbeats, to the overhead of the error control mechanism that depends in controlling the intra-frame update rate to limit the error propagation. The transmission overhead ratio is defined as the total number of bytes sent in intra-frames update scheme to the total number of bytes sent in MPRTP, to attain a given video quality represented by the average PSNR. In order to calculate the maximum overhead for MP-RTP, we used 3 paths. We varied the channel average packet loss rate for the primary path, path-0, while the channel average packet loss rates for the other paths, path-1 and path-2, were set to 0.2 and 0.1 respectively. Figure 9 shows the overhead ratio for average PSNR equal $35 \mathrm{~dB}$. In the single path retransmission case required an I-frame frequency of almost 1 per second for a video quality of around 35 dB. While with MP-RTP the same video quality can be attained with I-frame frequency of 1 per 3 seconds. The figure shows that the overhead of MP-RTP is less than that for the I-frames update scheme. The reason that the redundant retransmission mechanism implemented in MP-RTP is adaptive, in the sense that it only adds the retransmission overhead when there is loss in the video stream. 


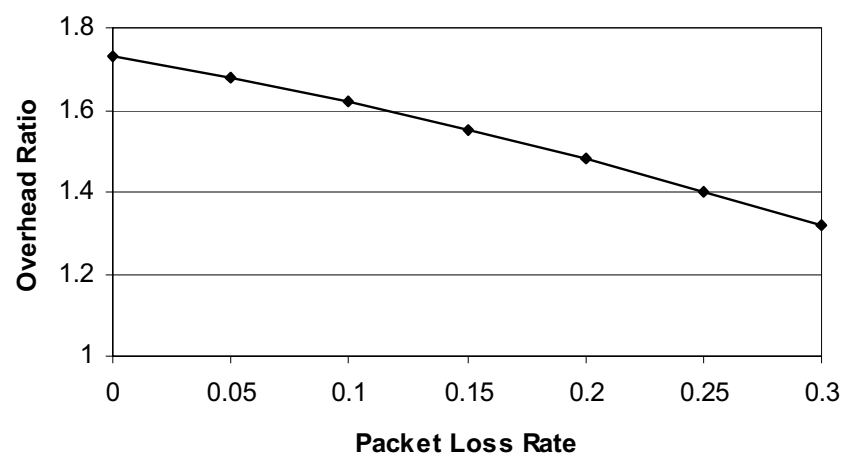

Figure 9. Overhead ratio versus average PLR

In addition, the degree of the overhead is proportional to the importance of the lost packets. Although heartbeat packets are periodically sent, they have less contribution to the overhead, as they are small in size compared to the size of video frames.

\subsection{Effect of Changing the Reliability Level for P-frames}

We examined the effect of varying the priority level of the P-frames, starting from the I-frame in the GOP up to the next I-frame, on the received video quality under different average packet loss rates. We used an Iframe update period equal 3 seconds. We set the sender to use two paths. We varied the channel average packet loss rate for the primary path, path- 0 , while we set the channel average packet loss rate for the path-1 to 0.2 . Figure 10 shows that the average PSNR versus the percent of $P$ frames set as high priority frames. It shows that quality will keep increasing as we protect more Pframes within the GOP but the increase rate becomes slower after around $50 \%$. This is partly because Pframes that are farther from an I-frame have fewer blocks that can be coded as a difference from the Iframe (motion-predicted), which implies that the dependence decreases. This result confirms with the analytical model presented in [21]. We repeated the experiment with different sequences and we got similar results. This suggests that protecting up to $50 \%$ of the P-frames within the GOP through redundant retransmission is enough to maintain the reconstructed video quality, as protecting more P-frames comes with a price of increasing the transmission overhead.

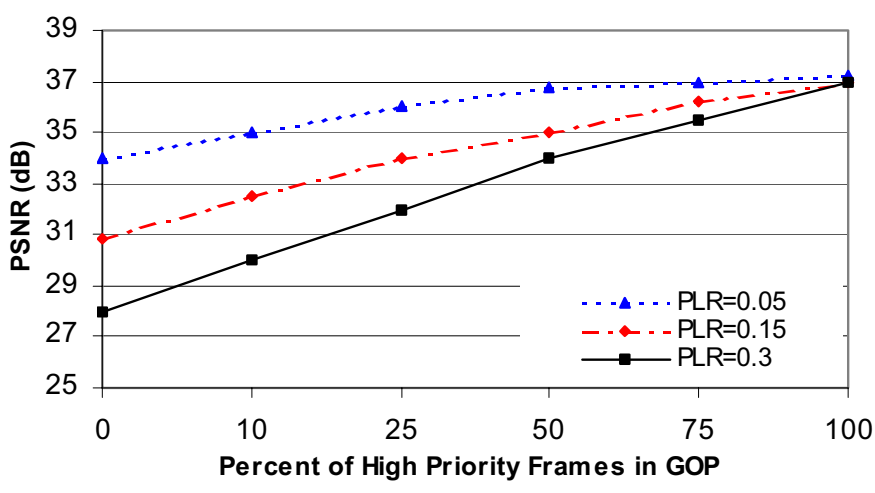

Figure 10. Average PSNR versus the percent high priority in GOP

\subsection{Comparison between MP-RTP and Layered Coding with Multi-path Transport}

In this experiment we compare MP-RTP with the error control mechanism, proposed in [11], that combines layered coding with multi-path transport. The mechanism protects the Base Layer (BL) packets through single path retransmission. We used a layered coder that generates a BL and one Enhancement Layer (EL). The BL is transmitted on path- 0 , while the EL is transmitted on path-1. Packets lost from the BL are retransmitted on EL path, while packets lost from the enhancement layer are not retransmitted. The channel packet loss rate for path- 1 is set to 0.2 , while we varied the channel packet loss rate for path-0. Figure 11 shows the sequence average PSNR versus the packet loss rate on path- 0 . We repeated the experiment with the same video sequence coded with a non-layered coder and transported with MP-RTP. We used the same network setup used for the layered video and we set path- 0 as the primary path. The figure shows that under high loss rate the quality of the layered video dropped more quickly than the non-layered video. The reason that the quality of the layered coded video depends on the conditions of the path that carries the base-layer packets. In addition, retransmitted packets may still be lost, due to the variable characteristics of the retransmission path. As far as the BL packets are lost, EL packets are discarded, even if they are received without errors. This causes the video quality to fluctuate with the error characteristics of the BL path. Thus, for interactive video, depending on single path retransmission to protect the $\mathrm{BL}$ packets is not enough to maintain the video quality under high loss rates. 


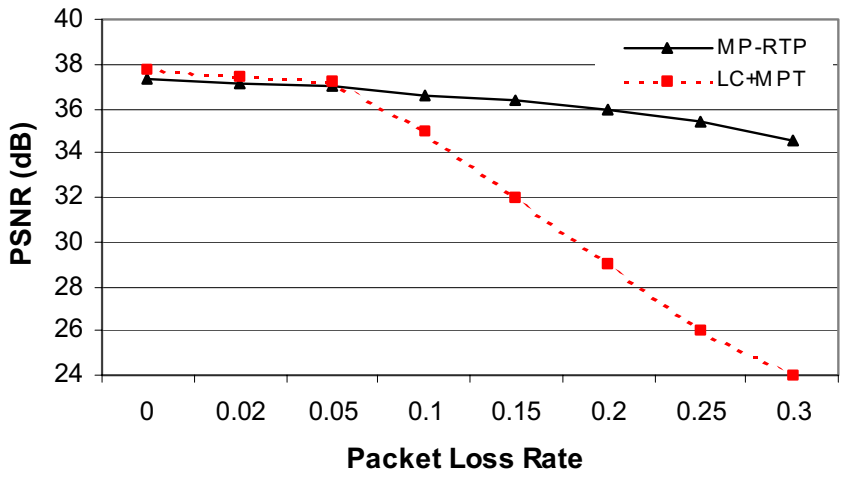

Figure 11. Comparison between MP-RTP and layered coding with multi-path transport

On the other hand, the redundant retransmission mechanism in MP-RTP, which benefits from the independent characteristics of the diverse paths between the sender and receiver, succeeds in maintaining the video quality regardless the packet loss rate.

\section{Conclusion}

Although encoding video using motioncompensated prediction achieves high compression efficiency, it suffers from the error propagation problem. Error due to packet losses propagate from reference frames to dependent frames, causing visual artifacts. This requires the transport mechanism to provide higher protection for reference frames than dependent frames. Interactive video complicates the problem by bounding the time available for the error control. To tackle these problems, we propose a mechanism to provide unequal error protection to data within the video stream according to their importance to the reconstructed video quality. The unequal error protection is realized through extending the classic retransmission based error control, with redundant retransmissions on diverse paths obtained by the adhoc routing protocols, in order to increase the probability that at least one of the retransmitted packets arrive at the receiver in less number of retransmissions. The degree of redundant retransmission depends on the reliability level required for the data within the retransmitted packet. A delayed constrained retransmission, based on the packet lifetime and estimate of the delay from the sender to receiver, is used to prevent re-transmitting expired packets. We implemented the proposed mechanism as an extension to RTP, refereed to as Multi Path - RTP (MP-RTP). Performance results show that the mechanism is able to provide a good quality for interactive video under different packet loss rates, with less transmission overhead than error control mechanisms that is based on controlling the intra-frame updates. In addition, MPRTP is shown to be more robust than error control schemes that combine layered coding with the path diversity in ad-hoc networks.

\section{Disclaimer}

The views and conclusions in this document are those of the authors and should not be interpreted as representing the official policies, either expressed or implied, of the Army Research laboratory or the U.S. Government.

\section{References}

1. N. Farber, K. Stuhlmuller, B.Girod, "Analysis of Error propagation in Hybrid Video Coding with Application to Error Resilience", Proc. IEEE Int. Conf. On Image Processing (ICIP), pp. 550-554, Oct. 1999.

2. Y. Wang, Q. Zhu, "Error Control and Concealment for Video Communications A Review", Proceedings of IEEE, vol. 86, no. 5, May 1998.

3. D. Wu, Y. Hou, W. Zhu, Y. Zhang, J. Peha, "Transporting Real-Time Video over the Internet: Challenges and Approaches", Proceedings of the IEEE, vol. 88, no. 12, pp. $1855-1875$, Dec. 2000.

4. J. Bolot and T. Turletti, "Experience with Control Mechanisms for Packet Video in the Internet", ACM Sigcomm Computer Communication Review, vol. 28, no. 1, pp. 4-15, Jan. 1998.

5. M. Zorzi, "Some Results on Error Control for BurstError Channels Under Delay Constraints", IEEE Transactions on Vehicular Technology, vol. 50, no. 1, Jan. 2001.

6. B. Dempsey, "Retransmission-Based Error Control for Continuous Media in Packet Switched Networks", Ph.D. thesis, University of Virginia, May 1994.

7. H. Liu, H. Ma, M. El Zarki, S. Gupta, "Error Control Schemes for Networks: An Overview", ACM Mobile Networks and Applications (MONET), vol. 2, no. 2, pp. 167182, Oct. 1997.

8. I. Rhee, R. Joshi, "Error Recovery using FEC an Retransmission", Dept. of Computer Science, North Carolina State University, Technical Report, TR-98-12, June 1998.

9. V. Goyal, "Multiple Description Coding: Compression Meets the Network", IEEE Signal Processing Magazine, vol. 18, pp. 74-93, Sept. 2001.

10. N. Gogate, D. Chung, S. Panwar, Y. Wang, "Supporting Image and Video Applications in a Multihop Radio Environment Using Path Diversity and Multiple Description Coding", IEEE Transactions on Circuits and Systems for Video Technology, vol. 12, no. 9, pp. 777-792, Sep. 2002.

11. S. Shunan, L. Shivendra, S. Panwar, Y. Wang, "Reliable Transmission of Video over Ad-hoc Networks Using Automatic Repeat Request and Multi-path Transport", 
ACM Sigmobile Computing and Communications Review, vol. 7, no. 1, pp. 59-61, Jan.2003.

12. B. Girod, N. Farber, "Feedback-Based Error Control for Mobile Video Transmission", Proceedings of the IEEE, vol. 87, no.10, Oct. 1999.

13. A. Nasipuri, S. Das, "On-Demand Multipath Routing for Mobile Ad-hoc Networks", IEEE International Conference on Computer Communications and Networks ICCCN, Oct. 1999.

14. S. Lee, M. Gerla, "Split Multipath Routing with Maximally Disjoint Paths in Ad-hoc Networks", IEEE International Conference on Communications ICC, 2001.

15. M. Pearlman, Z. Haas, P. Sholander, S. Tabrizi, "Alternate Path Routing in Mobile Ad-hoc Networks", IEEE Milcom, Oct. 2000.

16. ITU-T Recommendation, "H.263, in Video Coding for Low Bitrate Communication”, Sep. 1997.

17. H. Schulzrinne, S. Casner, R. Frederick, and V. Jacobson. "RTP: A Transport Protocol for Real-Time Applications", RFC 3550, Jul. 2003.

18. Opnet Simulation and Modeling Tool, www.opnet.com. 19. E. Gilbert, "Capacity of a burst-noise channel", Bell System Technical Journal, vol. 39, no. 9, pp. 1253-1265, Sep. 1960.

20. XviD MPEG-4 video codec, www.xvid.org

21. N. Feamster, H. Balakrishnan, Packet Loss Recovery for Streaming Video, International Packet Video Workshop, Pittsburgh, PA, Apr. 2002. 